\title{
First Do No Harm: Hamilton Health Sciences' Experience as First Hospital in the World To Fully Implement Medley ${ }^{\mathrm{TM}}$ Medication Safety System
}

\author{
Paul Faguy, Leslie Gauthier and Mark Duffet
}

$\mathrm{H}$ amilton Health Sciences (HHS) had a challenge on its hands. The hospital's intravenous (IV) infusion pumps were out-of-date and had to be replaced. As we began to investigate the options in 1998, we realized we had a choice: purchase updated versions of traditional technology or enter uncharted territory with new computerized "smart" technology that was still under development. The potential of the new IV infusion system was impressive but it wasn't yet proven and HHS would have to shoulder some of the growing pains as it was further developed.

The promised gains in medication safety persuaded us to choose innovation. In 2001, HHS, along with our sister hospital in Hamilton, St. Joseph's, became the first in the world to acquire and fully implement the Medley TM

\section{The Patient Safety Issue: When Healers Cause Unintentional Harm}

Until this year, health authorities did not have a wealth of Canadian data on medication errors. In May 2004, the first Canadian study to quantify the risk of inadvertent harm to hospital patients was published and its findings were shocking (Baker and Norton 2004).

The Canadian Adverse Events Study found that in the year 2000, Adverse Events (AEs) occurred in $7.5 \%$ of admissions to acute care hospitals in Canada, about one in 13 admissions. Although most patients recovered without permanent disability, AEs are causing significant permanent disability and death. The researchers estimated that AEs may be responsible for anywhere from 9,250 to 23,750 deaths a year.

... committee members were impressed with many of the system's advanced features but were particularly intrigued with the company's vision for safety software with unprecedented sophistication.

Medication Safety System, manufactured by ALARIS Medical Systems, Inc.

Three years later, it is clear the decision was the right one. Our clinicians say the change has revolutionized our delivery of medications and fluids, and has enhanced the quality of patient care on a number of levels. The impact on safety has been proven, through documentation of the prevention of hundreds of medication errors at the bedside. Our experience with the Medley system and the feedback we have provided have led the manufacturer to make numerous technology and procedural improvements that are now benefiting not only our hospitals but other hospitals using the system across North America.

In concert with our decision, in 2004, Canadian regulators, patient safety organizations and other hospitals more fully recognize the risks of medication error and the valuable role that technology can play in error prevention.
The study determined that events associated with drug or other fluid delivery were the second most common type of Adverse Event (AE), behind events associated with surgery.

Also in 2004, Health Canada issued a Notice to Hospitals, warning of the risks associated with intravenous infusion pumps (Health Canada 2004).

Between 1987 and March of 2003, Health Canada received reports of 425 separate incidents involving infusion pumps. Of the total, 23 resulted in death, 135 resulted in injury and 127 could potentially have led to death or injury. Of these, 20 of the deaths and all 135 of the injuries were caused or suspected to have been caused by the pump, either a problem with the technology or user error.

Health Canada recommended a number of safety steps to protect against infusion device problems and medication errors. One of the key recommendations was: "Users 
should consider purchasing pumps with safety features such as software that provides safeguards against dosing and infusion rate errors. These 'smart' pumps can incorporate hospital-defined protocols with built-in maximum allowable dose and infusion rates for specific patient types, or alarms to indicate when an infusion parameter is outside a pre-determined range."

Healthcare providers recognize that medication errors can occur anywhere in the chain of delivery that begins with the prescription order but mistakes earlier in the chain are often intercepted well before the medication reaches the patient. At the point of drug administration, errors have traditionally been more difficult to detect and clearly, the consequences of error more serious.

\section{The Promise of the New Technology}

Decision-making on the new IV technology rested with a multidisciplinary committee representing nursing, physicians, pharmacy, biomedical technology and human resources/hospital services.

When we first began exploring the options with ALARIS, committee members were impressed with many of the system's advanced features but were particularly intrigued with the company's vision for safety software with unprecedented sophistication. We decided to partner with the company to become the first in the world to implement the technology and ultimately to help refine the software. This partnership was also advantageous for ALARIS, in that Hamilton Health Sciences and St. Joseph's offers a full range of patient care services from adult critical care, to pediatrics and neonatal intensive care. Together, we have a total of 1564 beds and provide care to patients with all types of disease processes and injuries, throughout the life span.

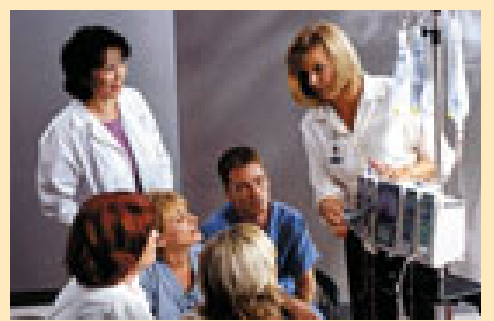

The infusion system can be programmed to deliver as many as four medications at one time
The Medley system is a modular platform that uses a point-ofcare-computer to integrate infusion, patient monitoring and a hospital's clinical best practice guidelines. The "smart"part of the infusion system resides in the PCU - known as the "brain". It can be programmed to deliver as many as four medications at one time, through modules that snap onto the main module.

The Guardrails ${ }^{\circledR}$ Safety Software, allows hospitals to configure care-specific profiles with pre-defined drug dose limits and other delivery parameters. It provides each infusion device with a database of information on medications given by continuous infusion in the hospital and it acts as a safety net for a nurse who may be poised to exceed or underdose the safe dosing limit in error.

The Guardrails were to be the foundation of this intelligent technology but as we discovered, implementation involved much more. A meticulously researched database of safe dosing limits, ongoing training and education and a culture of patient safety across hospital units and healthcare disciplines, were all required to make this new approach to medication safety truly successful.

\section{The First Step - Setting up the Medication Library}

When a clinician enters the Guardrails drug profiles, he or she is accessing the results of several months of extensive research and consultation.

All clinicians were involved in setting minimum and maximum doses for infusions to be used across the healthcare facility. Physicians, pharmacists and nurses from HHS and St. Joseph's collaborated to set standards for drug dosing units, concentrations, dose ranges and operating parameters for each of the patient units.

The process of creating this new medication library led our health professionals to take a critical look at past practices. Previously, there had been a number of different IV delivery systems and variation in medication standards used in different units and sites. It was a lengthy and complex process but the result was an unprecedented consensus and the uniform implementation of medication best practices across the hospital sites. At the same time, medication profiles were customized as appropriate for specific units. We currently have nine different unit profiles.

\section{Building and Maintaining the Safety Culture: Training}

In its Notice to Hospitals, Health Canada also recommends that all hospitals ensure that users are properly trained in the use of infusion devices.

Ongoing training and education have been integral to the effectiveness of the new medication safety system and to the building and maintenance of the patient safety culture. Since 2001, we have worked with the company to train nurses, pharmacists and educators on safe use of the pumps. Additional staff training is provided with each software upgrade and each user of the pump must demonstrate their competency with use of a skills checklist. 


\section{Staying Inside the Medication Safety Zone - How the System Works}

When a nurse begins to set up the IV through the programing device for a continuous infusion, he or she enters the Guardrails software and goes into the medication "library"customized for the specific unit, such as neonatal or adult intensive care. The nurse highlights the name of the drug to be administered, chooses the infusion mixture and enters the dose to be delivered. The device then calculates the flow rate required.

It is important to note that the system is set up to enhance, not replace, the individual clinician's judgment. If the nurse starts to program a dose that is above (or in some cases, below) the safe limit of that drug, the device will sound an alert and the question "Do you wish to proceed?" will appear on the device's screen. At this point, clinicians examine both their use of the device and their decision-making.

In many cases, the alert is prompted by a programming error, which, for example, might have led the nurse to program $200 \mathrm{~mL}$ per hour of a medication instead of 20

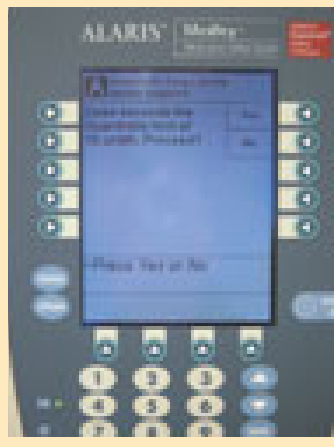

The alert can show the nurse a programming error has occurred $\mathrm{mL}$ per hour. These types of ten-fold dosing errors are quite common. The alert can show the nurse a programming error has occurred and it can be corrected quickly.

However, a review may well reveal that the numbers were punched correctly. Here's where the nurse begins to exercise further clinical judgment.

There are "soft Guardrails" and "hard Guardrails." Hard Guardrails are inviolable. For certain drugs, the system will not allow the operator to exceed the maximum under any circumstances. The pump simply cannot be programmed if any effort is made to go outside these hard limits, forcing the provider to change the dosage and re-program. However, soft Guardrails can be overridden.

The nurses have to make a conscious decision to override a Guardrail. In certain cases, exceeding the Guardrail is medically necessary, for example, when trying to resuscitate a patient in cardiac arrest or for a patient on morphine who has developed tolerance and needs a higher dose to control pain.

In other cases, the nurse knows that the programming was consistent with the original prescription and does not see any clinical reason to override a Guardrail. This should prompt the nurse to consult with other members of the healthcare team to determine if there was an ordering error or if the dose is appropriate for the clinical scenario.

\section{Impact: Safety Gains}

The smart infusion technology has led to quantifiable safety gains. Because the computerized database keeps a record of every Guardrail alert that occurs, we have a valuable storehouse of data on when Guardrails were exceeded and for which drugs on which units.

The hospital downloads and analyses this data regularly and gathers important information on how the Guardrails are working. A recent analysis shows that the guardrails prevented approximately 576 potentially significant errors from reaching patients in 2003. The Guardrail CQI (Continuous Quality Improvement) data also allows us to understand issues in the prescription and administration of our medications.

This CQI data capability is a powerful feature of the system. This data provides information on the near-misses averted by the system at the point of care. Clinicians may then use this information to assess current practices and take further steps to enhance safety.

If, for example, a particular Guardrail is consistently being overridden, the team may decide to change the drug profile or to provide additional training to the care providers. All this information leads to periodic adjustments to the medication library and to new training approaches.

\section{Impact: Quality of Patient Care}

Some of the positive results are less quantifiable but nonetheless critical. Clinicians say the quality of patient care has improved. Having a smart infusion device on their side helps care providers infuse medications safely and efficiently, despite the inevitable distractions which can arise in busy units with many patients to care for. The compact modular setup, with multiple channels, takes up less space, weighs less than other devices on the market, and gives nurses greater ease of movement when caring for patients.

Before smart pumps, the health professional spent a good deal of time doing complicated and time-consuming calculations to set up infusion rates. The automatic control frees up the clinician to concentrate even more on patient safety and patient care.

\section{Impact: Greater Collaboration}

From the creation of the drug database, to the continuous updating of the system in response to identified concerns, 
the Medley system has fostered even greater collaboration among all healthcare groups at HHS and St. Joseph's. Pharmacists, nurses, physicians and other team members are all involved in the mission to bring safety to a higher level.

For each software upgrade, there is extensive consultation and collaboration and this has given clinicians a renewed focus on working together to constantly improve practice.

\section{HHS Contributions to Evolving Medley System}

As the first customer in the world, HHS was an important partner in the development of some of the features of the Medley system that exists today. The company has frequently streamlined the technology and the software to make it easier to use and truly responsive to the requirements at the bedside. Our system has undergone seven upgrades over the past three years, largely in response to our experience on the wards.

One key change that HHS inspired, involved navigation of the Guardrails software. In the first version, the Guardrail feature was under "options" and nurses had to press seven or eight keys to access the drug safety system. As a result, some users were not taking the time to activate the safety features of the devices. It soon became clear that to truly improve safety, the Guardrails could not be optional. The change was made and the Guardrails now come up immediately on the screen as the programing begins and their use is mandatory.

Another example: nurses in the Neonatal Unit urged the company to work on a new syringe module to permit more precise delivery of potent drugs and lipids for infants. The Medley Syringe Pump Module was developed and has been in use in the Neonatal Unit at HHS since this summer.

\section{Lessons Learned}

Hamilton Health Sciences has had a unique experience with the Medley system and the lessons we learned are paving the way for other healthcare facilities to bring in the system more rapidly and efficiently. The system has recently been acquired by other Canadian hospitals, including the Health Care Corporation of St. John's in Newfoundland, St. Joseph's Health Care in London and the Markham Stouffville Hospital.

Our experience has demonstrated that hospitals instituting such a system need to do the cultural work around safety and to understand that the change is not simply a project; it is a complete overhaul of all medication processes. It is critical to establish a process for getting team consensus around how to run your medications and a process for investigating incidents. There must be an infrastructure to support this system beyond implementation, so hospitals can continually learn from failures and make safety enhancements.

There have been challenges. Our current technology makes the process of downloading data and updating the database very time consuming as it must be done one device at a time. Newer technology provides a wireless system that immediately sends the data to a central server, which allows for faster changes.

Analysis of our CQI data has shown that in some cases the nurses are not reprograming the device when the alerts occur. Although some of the overrides are clinically indicated, others are not and HHS is continuing its training and education of clinicians to ensure that all decisions to override Guardrail alerts are informed by prudent clinical judgment.

\section{Further Enhancements}

Hamilton Health Sciences plans to expand its partnership with ALARIS in the implementation of other innovations in the Medley system and to share this knowledge more widely with other healthcare facilities in Canada. One example is in the area of Patient-Controlled Analgesia (PCA). HHS is in the process of purchasing new PCA pumps. Potentially, HHS will soon begin using new ALARIS PCA pumps, which will allow patients to manage their own pain through pushing a button on their infusion device. The Medley PCA module will have the same protection against potentially harmful errors.

\section{About the Authors}

Paul Faguy is Vice President Human Resources and Hospital Services at Hamilton Health Sciences.

Leslie Gauthier is a Clinical Manager in the Cardiac and Vascular Program at Hamilton Health Sciences.

Mark Duffet is a pharmacist at Hamilton Health Sciences

\section{References}

1. G. Ross Baker, Peter G. Norton et al. The Canadian Adverse Events Study: the incidence of adverse events among hospital patients in Canada. CMAJ, May 25 2004: 170(11), 1678-1686.

2. Health Canada. Health Products and Food Branch. Notice to Hospitals. Health Canada Endorsed Important Safety Information on INFUSION PUMPS. April 16, 2004. 